\title{
Mobile Station-Controlled Handover Scheme in Mobile WiMAX: Case Study
}

\author{
Emran Hassan Al-Saleh \\ Computer Science Department \\ Zarqa University \\ Zarqa, Jordan
}

\author{
Ghassan Samara \\ Internet Technology Department, Faculty of \\ Information Technology \\ Zarqa University, Zarqa, Jordan
}

\begin{abstract}
Mobility in WiMAX system is one of the most important problems in computer networks, especially when a mobile station (MS) tours and be handovered from one base station (BS) to another, Mobile WiMAX is a broadband system that is capable of delivering services such as voice, data and video. In this paper, fast and simple scheme of handover (HO) in Mobile WiMAX network will be presented using pathloss, received signal strength, angles of divergence which leads to better and fast HO scenarios. Simulation studies show low latency in the handover, better throughput and less packet loss ratio during the $\mathrm{HO}$ process.
\end{abstract}

\section{Keywords}

Handover, Mobile WiMAX, Distance Estimation, Lookahead, Angle of Divergence.

\section{INTRODUCTION}

It goes without saying, with the development of telecommunications technology and development in the science of wireless communication, especially in cellular networks with attractive features such as high data rate, extended area coverage, spectral efficiency and low cost are increasing the deployment of Mobile WiMAX (IEEE 802.16e) networks. However, the improvement HO process remains an important area in research. $\mathrm{HO}$ is the process of transferring an ongoing call or data session from one channel to another through the core network. It must be executed fast, without causing any call drop, without exhaustion much of the network resources, and without causing any throughput degradation. With different recommendations that were formed in Mobile WiMAX standard[1] and WiMAX Forum documents[2] with respect to the parameters to be used types of mobility, a large number of various approaches toward performing $\mathrm{HO}$ have been suggested in the literature. In the next section a brief review of these will be provided. The current paper discussed a case study of hard HO scheme which called MS-controlled fast handover[3] in Mobile WiMAX.

In this scheme, the MS depending on estimating its current distances from its NBSs by using their RSS and performing a suitable lookahead algorithm for selecting its TBS. Actually, the MS can itself achieve its need of a HO with many factors such as the knowledge of the RSS of its NBSs and SBS, and the knowledge of its own absolute velocity, then it can select the TBS, and finally request the SBS to handing it over to its selected TBS. This technique of RSS-based distance estimation followed bya suitable lookahead was developed in connection with algorithm use in Mobile Ad-Hoc Networks (MANET), namely, a Modified Distance Vector Routing (MDVR) algorithm[4].The main benefits of such simple MScontrolled HO techniques in Mobile WiMAX that is enhanced the scalability of Mobile WiMAX networks by distributing of the $\mathrm{HO}$ work of each BS to the large number of MSs being served by it. As a matter of fact, the selection of TBS by MS based on two criteria: (i) the bandwidth and quality of service (QoS) (ii) the highest relative vel00ocity with respect to the MS. So, any NBS do not provided QoS-BW requirement or do not show progressive motion with MS are not even considered as a TBS.

From the previous, with compared this scheme with other proposed HO schemes in Mobile WiMAX, by reducing the scanning and ranging activities so the $\mathrm{HO}$ delay is reduced and improving the expected call drop performance and throughput degradation.

The rest of paper is organized as follows. A briefly reviewed for the procedure of IEEE802.16e HHO and the related work of the HO research in section 2. In Section 3 explained the precept and implementation methodology of the MS-controlled HO scheme. The simulation steps present in Section 4. In section 5 presented simulation details and the numerical results that have been obtained. Finally, the conclusions drawn in Section 6.

\section{HHO IN MOBILE WIMAX AND RELATED WORK}

The standard of Mobile WiMAX supported three types of HO procedures, namely, the Fast Base Station Switching (FBSS) and the Macro Diversity HO (MDHO). Among of these types the HHO is the default and the most commonly used procedure. The HHO procedure[1] in Mobile WiMAX contains two main phases, the first phase is the Network Topology Acquisition Phase (NTAP), the second is the Actual Handover Phase (AHOP). In Mobile WiMAX, the HO process is excited when the received signal strength by the MS from its SBS drops below a particular threshold level. The gathering information about the network topology by the MS and the SBS with help of backhaul network achieved in NTAP before the actual HO decision is made. The SBS broadcasts information about the state of NBSs, their respective IDs, uplink and downlink channel descriptors (UCD and DCD) etc., by using the MOB_NBR-ADV message. The MS performs scanning and ranging activities, based on these information, for all NBSs regardless of QoS and BW of NBSs and the direction of MS movement before selected the appropriate TBS, with the help of SBS for a potential HO activity. By exchanged several MAC management messages between the MS and SBS to complete whole process. In AHOP, the MS send a Mobile Handover Indication (MOB_HO-IND) message to its SBS to terminates its connection with it after selection the TBS. Next, the MS make a series of MAC management procedures such as synchronization, ranging, authorization and registration, then the MS becomes connected with new SBS. A detailed depiction of the HO procedure can be found in [1].In 
the familiar mobile WiMAX handover procedure, during NTAP, the MS performs excessive scanning activities for all the NBSs, in which all the data transmission will be paused through these activities. Throughput degradation and delay may be caused by unnecessary scanning activities, which affect the system efficiency. On the other hand, the AHOP suffers from lengthy gap for inter-HO because of the wide network reentry activities of an MS [5]. Most 802.16e HHO research recently has concentrated mostly on attempts to decrease the disruptive effects of these constraints. The schemes proposed in [6] and [7] concentrate on minimizing the disruptive effects on scanning of Mobile WiMAX channel activities in case of various types of traffic and noise levels during HO. Schemes proposed in [8] and [9] work on prediction of TBSs before scanning and ranging activities based on different factors such as BS's coverage, required QoS and bandwidth for $\mathrm{HO}$, etc. In [10] the scheme proposed to reducing handover latency by dereliction the gap of inter-HO during the AHOP. In a cross-layer HO scheme proposed in [11], the MS using the signal strengths of the BSs to prediction the mobility. The total HO delay here was reduced by initiating the activities of layer-3 prior the activities of layer-2. So, this scheme did not reduce the MAC HO time.

It should be noted that, the most of these proposed HO schemes in Mobile WiMAX controlled by the SBS with possible assistance from the MS, only the exception of them, the cross-layer HO scheme [11] which is the MS initiate the HO activities. So, the schemes controlled by SBS, creates the important issue of scalability owing to redundant load on the SBS. But in MS-controlled HO scheme, the MSs can determine where themselves, with their respective TBSs (new SBSs) and then request current SBS for performing the actual HO process, via the backbone of network, thus may provide a better alternative. In the MS-controlled HO [12], the MS can estimate of its current distance approximately from any NBS by using the measured value of RSS in a suitable pathloss formula. The MS make sure of the need of the HO through monitoring the RSS of the SBS periodically, and then starts scanning only those NBSs, which have been chosen as a potential TBS PTBS). With a many scanning cycles, after distance estimates of the NBSs, the MS select the NBS which it has the highest relative velocity, then requests the SBS to hand it over to this selected TBS.

\section{MS-CONTROLLED FAST HHO SCHEME}

To describe the MS-controlled fast HHO scheme, firstly, it will be explain the various ideas behind this scheme. At any time, The MS can approximately estimate its present distance from any base station (service or neighbor) by measuring the RSS received from the concerned base station and using this information of RSS in a suitable pathloss formula[13]. This parameter of RSS indicator (RSSI) used in familiar Mobile WiMAX HO framework to reduce the effect of noise and fading and actually obtained after some filtering of the received carrier signal followed by computing its logarithm. However, in this scheme, the received carrier signal as the RSS only after a suitable signal processing, and before computation of the logarithm is used. The idea from using this RSS for distance estimation has also been investigated in[14] for use in localization in WIMAX networks, and this study produced a new empirical pathloss formula to establish RSS-based distance estimation as applicable.

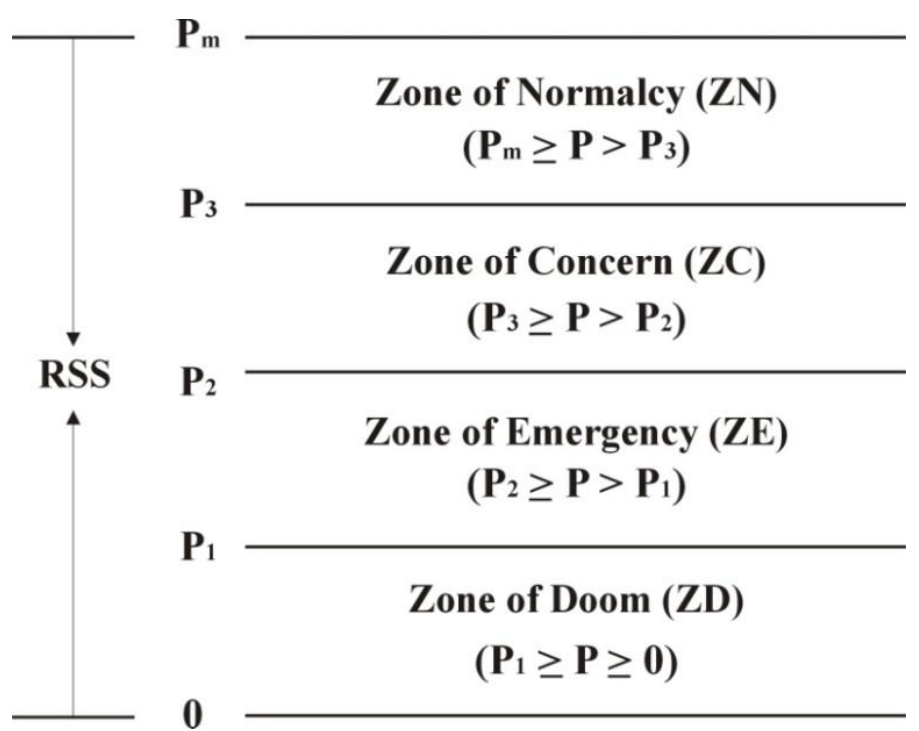

Fig 1: Zones based on RSS levels

In order to manage the entire process of handover in this scheme efficiently, it will be explain the concept of RSS power based zones. By dividing the RSS power $\mathrm{P}$ into the dynamic range $[0, \mathrm{Pm}]$, that the $\mathrm{MS}$ can receive from its $\mathrm{SBS}$, into three different levels P1, P2, and P3, the value of $\mathrm{P} 1<\mathrm{P} 2$ and $\mathrm{P} 2<$ $\mathrm{P} 3$, the MS produces four imaginary zones as shown in Figure 1. The name of zones is the Zone of Normalcy $(\mathrm{ZN})$, the Zone of Concern (ZC), the Zone of Emergency (ZE) and the Zone of Doom (ZD). Each zone corresponds to RSS powers in the range $[0, P m]$. The $\mathrm{ZN}$ corresponds to $\mathrm{RSS}$ power $\mathrm{Pm} \geq \mathrm{P}>\mathrm{P}$, $\mathrm{ZC}$ corresponds to RSS power $\mathrm{P} 3 \geq \mathrm{P}>\mathrm{P} 2$, $\mathrm{ZE}$ correspond to $\mathrm{P} 2 \geq \mathrm{P}>\mathrm{P} 1$ and $\mathrm{ZD}$ correspond to $\mathrm{P} 1 \geq \mathrm{P}$.

The SBS broadcasts MOB_NBR-ADV message to the MS [1], then the MS periodically monitors the RSS power of its SBS via it for identifying the zone it is currently in. In the $\mathrm{ZN}$, the needed of handover activity is very little except the periodic monitoring of the MOB_NBR-ADV message broadcasts. On the other hand, all handover-related activities, including the activities executed by SBS and network after selecting the TBS should be completed before MS enter the ZD. This final condition is purposed to avoid excessive packet losses, increase the throughput or avoid call drops, which may occur because of the very poor RSS in the ZD. From take a view on each zone, each zone can define by a lower and upper threshold, such as the ZD which defines the zone where the RSS threatens to drop the MSs at the lowest modulation scheme (typically $1 / 2$ rate QPSK), and defines the upper threshold P1 of ZD that is also the lower threshold of ZE. Similarly, the lower threshold of ZN defined as P3, may be taken to be the receiver's sensitivity at the highest modulation scheme (typically 5/6 rate 64 -QAM) or other highest modulation schemes to suit the operational requirements of the network operator. The lower thresholds P1 of ZE and P2 of ZC chosen to divide the interval between $\mathrm{P} 1$ and $\mathrm{P} 3$ into two parts based on the operational considerations of the network operator.

It is clear that, by helping this concept of four zones, the MS can perform its all handover-related activities at the right times and in the right sequence, and the MS can completes a good part of the handover-related activities even before the SBS reaches the pre-defined handover threshold level. 
At the same time, not only the RSS using by MS with SBS to select the zones, but also using for distance estimation of NBS to select AOD which is known lookahead technique which the MS performs it for controlling the $\mathrm{HO}$ process. By using the distance estimation and lookahead-based handover method, the MS performs a lookahead by estimating the angle of divergence (AOD) of each NBS with consideration to its own direction of motion. Figure 2 explains the precept of AOD of the MS and its NBS. If assumed that the MS moving along the direct line path AdC, the MS is currently existed at $d$ and the NBS is existed at B. The AOD of the MS with its NBS B is the angle $\mathrm{CdB}$, which is included between the direction of the MS linear motion and the line connecting the MS with the NBS. It is clearly, whenever the AOD of the MS with its NBS close to zero degree, whenever the MS movement is fastest towards its NBS. For explaining the AOD-based TBS lookahead scheme, the scenario illustrated in Figure 3 take as example, where the MS has six NBSs, A, C, D andE , clustered around its SBS S, and the MS is moving along the straight line XY.

How the MS chooses its TBS may be now explained as follows:

Step1: The MS receives high RSS power from its SBS when it stay in the $\mathrm{ZN}$, where the range of power between $\mathrm{Pm} \geq \mathrm{P}>$ P3. The MS creates a set $\{A, B, C, D, E, F\}$ of its NBSs by monitoring the periodic message MOB_NBR-ADV broadcasts by SBS S. In addition of this, may be add the current load BS or NBS which do not have sufficient QoS-BW features to excluded the NBS which is highly overloaded and thus do not have ability to become a TBS.

Step2: After leaving the $\mathrm{ZN}$, when the MS enters the ZC, it begins receiving a power $\mathrm{P}$ where the range of it between $\mathrm{P} 3$ $\geq \mathrm{P}>\mathrm{P} 2$ from the SBS, which is still much higher than the Minimum Acceptable signal Level (MASL)but less than normal power. So, in expectation of the potential need for a handover, the MS begins preparing itself for a handover activity. Thus, for initiating the scanning process of all NBSs, the MS sends a MOB_SCN-REQ message to its SBS. Up to receiving the MOB_SCN-RSP message from its SBS, the MS scans each NBS at every T second interval. The values of number of Scanning (NS) and T are chosen based on many factors like the current velocity of the MS, the number of NBSs, etc. Then, the MS keeps measuring the RSS from the SBS in order to know which zone currently it is in. The MS initiates two sequential scanning cycles at the time moments $t 1$ and $\mathrm{t} 2$ where $\mathrm{t} 2=\mathrm{t} 1+\mathrm{T}$ seconds. In Figure 3 , the MS is existing at points $\mathrm{x}$ and $\mathrm{y}$, respectively on the red line of its motion, at these two time moments. So, at the point $\mathrm{x}$, the MS scans the list of all NBSs A, B, C, D, E and F to get the RSSs from them for estimating their respective current distances $\mathrm{dA}$, $\mathrm{dB}, \mathrm{dC}, \mathrm{dE}$ and $\mathrm{dF}$ respectively from it. Next, after the suitably chosen interval of time T seconds, when the MS reach at the point $y$ on its line of motion, the MS begins a second scanning cycle for the all NBSs, or less if there RSS from any NBS became below the MASL, to estimate the changed distances $\mathrm{dA}^{\prime}, \mathrm{dB}^{\prime}, \mathrm{dC}^{\prime}, \mathrm{dE}^{\prime}$ and $\mathrm{dF}^{\prime}$ from it.

Thus, pair of distance for each NBS have been obtained after the MS performs two scanning cycles which observed in Figure 3. these pairs are (Ax, Ay) for $\mathrm{A},(\mathrm{Bx}, \mathrm{By})$ for $\mathrm{B},(\mathrm{Cx}$, $\mathrm{Cy}$ ) for $\mathrm{C},(\mathrm{Dx}, \mathrm{Dy})$ for $\mathrm{D},(\mathrm{Ex}, \mathrm{Ey})$ for $\mathrm{E}$ and $(\mathrm{Fx}, \mathrm{Fy})$ for $\mathrm{F}$ and their measures are $\left(\mathrm{dA}, \mathrm{dA}^{\prime}\right),\left(\mathrm{dB}, \mathrm{dB}^{\prime}\right),\left(\mathrm{dC}, \mathrm{dC}^{\prime}\right),(\mathrm{dD}$, $\left.\mathrm{dD}^{\prime}\right),\left(\mathrm{dE}, \mathrm{dE}^{\prime}\right)$ and $\left(\mathrm{dF}, \mathrm{dF}^{\prime}\right)$ respectively. In addition of each pair and their measures, a triangle has been figuration for each NBS such as $\boldsymbol{\Delta} \mathrm{xAy}$ for $\mathrm{A}, \boldsymbol{\Delta} \mathrm{xBy}$ for $\mathrm{B}, \boldsymbol{\Delta} \mathrm{xCy}$ for $\mathrm{C}, \boldsymbol{\Delta} \mathrm{xDy}$ for $\mathrm{D}, \boldsymbol{\Delta} \mathrm{xEy}$ for $\mathrm{E}$ and $\boldsymbol{\Delta} \mathrm{xFy}$ for $\mathrm{F}$, and all triangles has the same common side (base) xy which located on the line of motion of the MS.

The assumption of all triangles with same base xy to calculate the "angle of divergence" AOD (e.g., angle Cxy) with each NBS on each triangle and take the QoS for each NBS to determine the PTBSs.

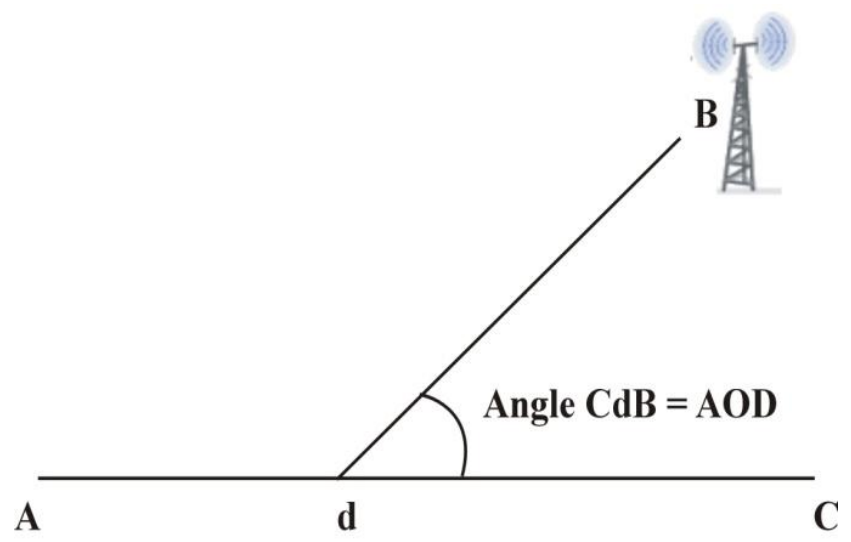

\section{AdC $=$ The direction of motion for the MS}

\section{Fig 2: AOD of an MS Moving with its NBS Precept}

The AOD value $\theta\left(0^{\circ} \leq \theta \leq 180^{\circ}\right)$, which is different for various NBSs, distinguishes the direction of motion of the MS which belongs to each NBSs as detailed in Table 1. With consideration to the table, the concept of a triangle itself disappears for the special values of $\theta=0^{\circ}$ and $\theta=180^{\circ}$.

Thus, it is obvious from the previous table, that the PTBS which have the lowest value of AOD $\theta$ will be offer the strongest RSS to the MS in the near future as it will move to it. As a result, this PTBS will offer the strongest signal to the MS and should be selected as the TBS. However, to accomplish this lookahead, it must be discovered the minimum value of $\theta$ to identify the PTBS. This problem solved with the following two observations:

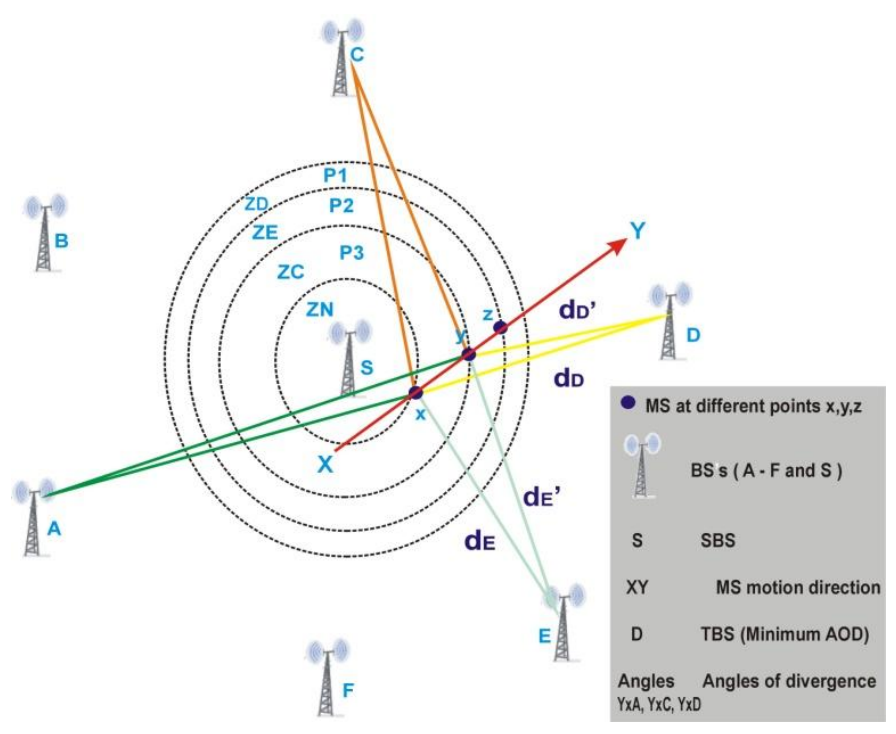

Fig 3: Distance Estimation with AOD based Lookahead scheme 
Table 1. Angles and their Description of MSs Motion

\begin{tabular}{|c|c|}
\hline Value of $\boldsymbol{\theta}$ & $\begin{array}{c}\text { Description of the MS motion with respect } \\
\text { to PTBS }\end{array}$ \\
\hline $0^{\circ}$ & $\begin{array}{c}\text { The MS is moving exactly towards the } \\
\text { PTBS, which means the MS will have the } \\
\text { highest possible forward movement } \\
\text { towards the PTBS. }\end{array}$ \\
\hline $0^{\circ} \leq \theta \leq 90^{\circ}$ & $\begin{array}{l}\text { The MS is moving towards the PTBS but } \\
\text { its forward movement towards the PTBS } \\
\text { will be less than the highest possible } \\
\text { value, which happens at } \theta=0 \text {. }\end{array}$ \\
\hline $90^{\circ}$ & $\begin{array}{c}\text { The MS movement is tangential and } \\
\text { cannot distinguish as either progressive } \\
\text { or regressive with respect to PTBS. }\end{array}$ \\
\hline $\begin{array}{c}\text { The movement of MS is away from } \\
\text { PTBS but its regressive movement will } \\
\text { be less than the highest possible value, } \\
\text { which happens at } \theta=180 \text {. }\end{array}$ \\
\hline $180^{\circ}$ & $\begin{array}{c}\text { The MS is moving exactly away from the } \\
\text { PTBS, which means the MS will have the } \\
\text { highest possible backward movement } \\
\text { away from the PTBS. }\end{array}$ \\
\hline
\end{tabular}

- The lengths of All sides in each triangle are known, e.g. $\Delta$ Axy, while the lengths of the sides Ax and Ay have been estimated through scanning and RSS measurement, and the length of third common side xy can be computed as the:

$$
\text { Length }(x y)=v T
$$

where $\mathrm{v}$ is the average of the MS velocity during the time $\mathrm{T}$.

- In trigonometry, in accordance with the "Law of Cosines", cosine of any angle of a triangle can be computed, if all three sides of it are known. In each of the four triangles $\boldsymbol{\Delta}$ Cxy , $\boldsymbol{\Delta}$ Dxy , $\boldsymbol{\Delta}$ Exy and $\Delta$ Fxy in Figure 3.4 which have the same base xy, the cosine of their angles, namely, $\operatorname{Cos} \theta \mathrm{C}, \operatorname{Cos} \theta \mathrm{D}$, $\cos \theta \mathrm{E}$ and $\cos \theta \mathrm{F}$, if the BS D take as example and applying the Law of Cosines, we have:

$\operatorname{Cos} \theta \mathrm{D}=\left\{(\mathrm{Dx})^{2}+(\mathrm{xy})^{2}-(\mathrm{Dy})^{2}\right\} /\{2(\mathrm{Dx})(\mathrm{xy})\} \ldots(2)$

The PTBS, which belong to the minimum angle among these four angles, $\theta \mathrm{C}, \theta \mathrm{D}, \theta \mathrm{E}$ and $\theta \mathrm{F}$, it will be have the highest value for the cosine of its angle. The Figure 3 shows that the angle $\theta \mathrm{D}$ is the smallest angle among these angles, so the computation of $\cos \theta$ for each angle will detect that $\cos \theta \mathrm{D}$ is the largest among them and for this reason $\mathrm{D}$ should be chosen as the TBS.

Thus, by finding the cosine AOD of the PTBSs and make a comparison among them, the MS can select the TBS which have a least value of AOD from all the PTBSs. However, if there are two PTBSs that have closely AOD values with respect $t$ each other, then the MS does not make the final selection of the TBS at this time and requires to check at last time. Instead, the MS selects two PTBSs which called Candidate TBSs (CTBS). The two BS must have the largest values of $\cos \theta$, must almost equal values, must show a progressive movement $\left(0^{\circ} \leq \theta \leq 90^{\circ}\right)$ and must have a level of signal greater than the MASL.

Step3: Upon completion of the $\mathrm{ZC}$ and reaching to $\mathrm{ZE}\left(\mathrm{P}_{2} \geq\right.$ $\mathrm{P}>\mathrm{P}_{1}$ ), the MS sends MOB_HO-IND message [4] to the SBS for executing an urgent handover by passing the ID of the selected TBS D. AS mentioned before, all HO process should be completed before the MS enters the ZD to avoid excessive packet loss or call drop because of the poor of RSS. However, in the case of selected two closely contesting PTBSs are selected as CTBSs in the ZC, it is clear that some addition delay may be occur in the ZE. So, in that case, in order to execute the final selection of the TBS between the two CTBSs, the MS executes the final pair of scanning repetitions for CTBS1 and CTBS2 at the point $\mathrm{z}$ in Figure 3. One from two CTBSs are selected based on if it shows both a progressive movement (compared with its previous distance), QoS is greater than another, and a level of signal greater than MASL. The flowchart of the AOD-based TBS lookahead scheme shows in Figure 4 where contains the functions implementing of the three main steps in that scheme.

\section{SIMULATION STEPS}

In this search, the MATLAB software version R2013b have been used for our simulations because of its easy to using in node deployment and network setup.

\subsection{Methodology}

- A service area ( $3500 \mathrm{~m}$ x $4000 \mathrm{~m}$ ) terrain for WiMAX infrastructure shows in Figure 5.These area divided into many cells, each cell have a set of mobile nodes, and each mobile node communicate with each other and moving randomly in some direction with some mobility rate.

- Deploy this topology and select the SBS S which provide the services to MS and it will be handovered to the NBS B.

- To choose the new best TBS an efficient form of lookahead technique is used based on distance estimation and AOD concept.

- $\quad$ The effect of noise is also considered to create a real time environment. So, there is an delay occurs in the form of noise.

- The constant bit rate (CBR) is chosen as the traffic type.

- Keep on increasing the speed of MSs in each simulation. The important simulation parameters listed in Table 2 according to the WiMAX forum specifications [16].

\subsection{HO Delay}

Here an assumption was made that a single MS have used to select the latency of HO. The red point represents the beginning of the movement and the green point represents the end of the movement for MS as it depicts in Figure 5. The nature of traffic used in the simulation is constant bit rate (CBR). According for our simulation model, one MS initially controlled by its SBS, and move from red to green point between the BSs, then perform a $\mathrm{HO}$ whenever needed during the simulation. A Mobility model [15] was used to model movements of the MS during simulations for various speeds from $5 \mathrm{Km} / \mathrm{hr}$ to $120 \mathrm{Km} / \mathrm{hr}$ [11]. The path loss exponent in free space [13] is used to calculate the pathloss effects during simulation.

\subsection{Throughput (Th)}

Throughput is a measure of how many units of information or number of packets successfully delivered through the network in a given amount of time. It is measured in terms packets/second during the HO process. The amount of throughput should be high or else it affects every service in 
Mobile WiMAX. Equation 3 shows how to calculate throughput[17].

$$
T h=\frac{\sum_{i} \text { Packets Delivered }}{\left.\left(\sum_{i} \text { Packets Arrival }- \text { Packet start time }\right)_{i}\right)}
$$

where $i$ is the number of MSs on the service area.

Table 2. Key Simulation Parameters

\begin{tabular}{|c|c|}
\hline Parameters & Values \\
\hline Service Area & 3500m x 4000m \\
\hline Type of Technology & IEEE 802.16e \\
\hline Number of BSs & 7 \\
\hline Number of MSs & 21 \\
\hline Number of cells & 7 \\
\hline BS max transmit power & $20 \mathrm{~W}$ \\
\hline MS max transmit power & $200 \mathrm{~mW}$ \\
\hline Wave Length & $10 \mathrm{e}-2$ \\
\hline Loss Factor & 1.5 \\
\hline FFT Size & 1024 \\
\hline MAC Address & Auto Assigned \\
\hline MAC Propagation delay & $1 \mu \mathrm{s}$ \\
\hline Frequency & $2.4 \mathrm{GHz}$ \\
\hline Number of Bits per packet & $1024 \mathrm{Bits}$ \\
\hline Time taken to travel & 20 \\
\hline Default Frame Length & $20 \mathrm{~ms}$ \\
\hline BS Antenna Height & $15 \mathrm{~m}$ \\
\hline QPSK Encoding Rate & 0.5 \\
\hline BS Link Propagation Delay & $1 \mathrm{~ms}$ \\
\hline Scan Interleaving Interval & $6 \mathrm{frames}$ \\
\hline MS's Movement Speed & $5 \mathrm{Km} / \mathrm{hr}-120 \mathrm{Km} / \mathrm{hr}$ \\
\hline
\end{tabular}

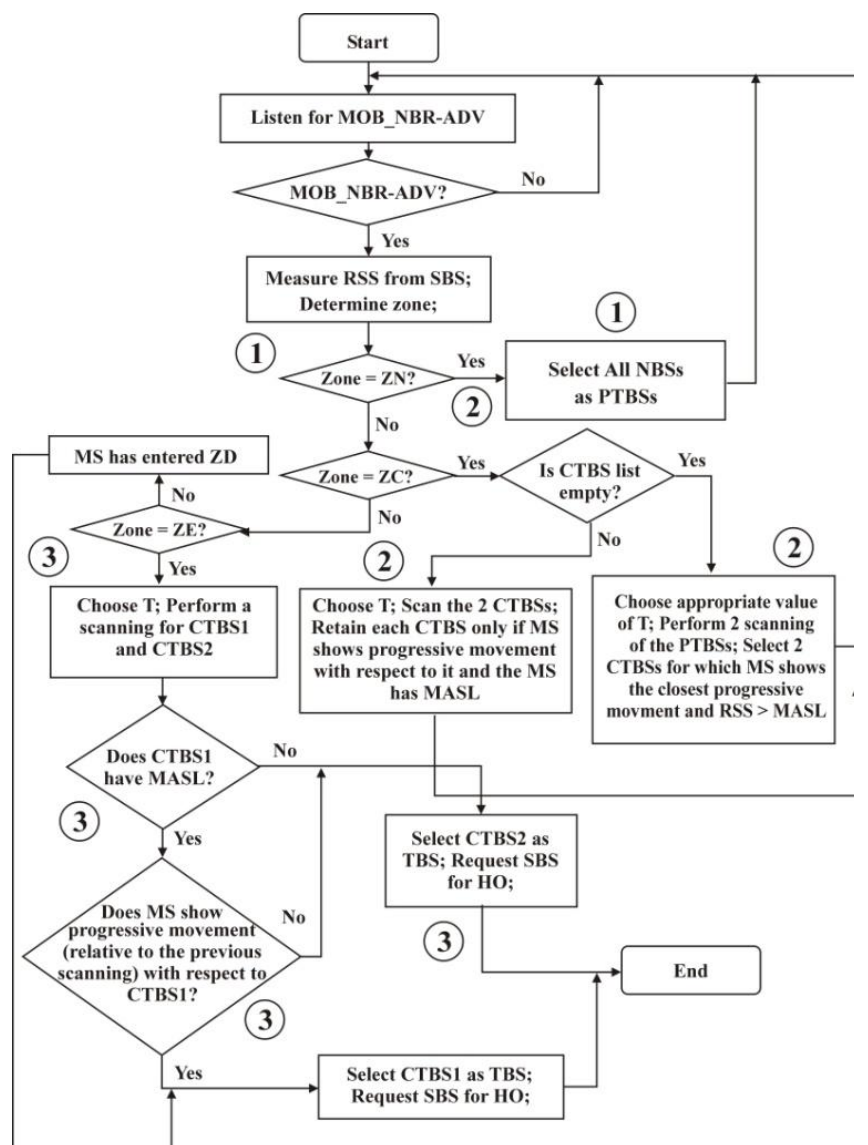

Fig 4:Flowchart of the AOD-based Fast MAC-Layer Handover Scheme

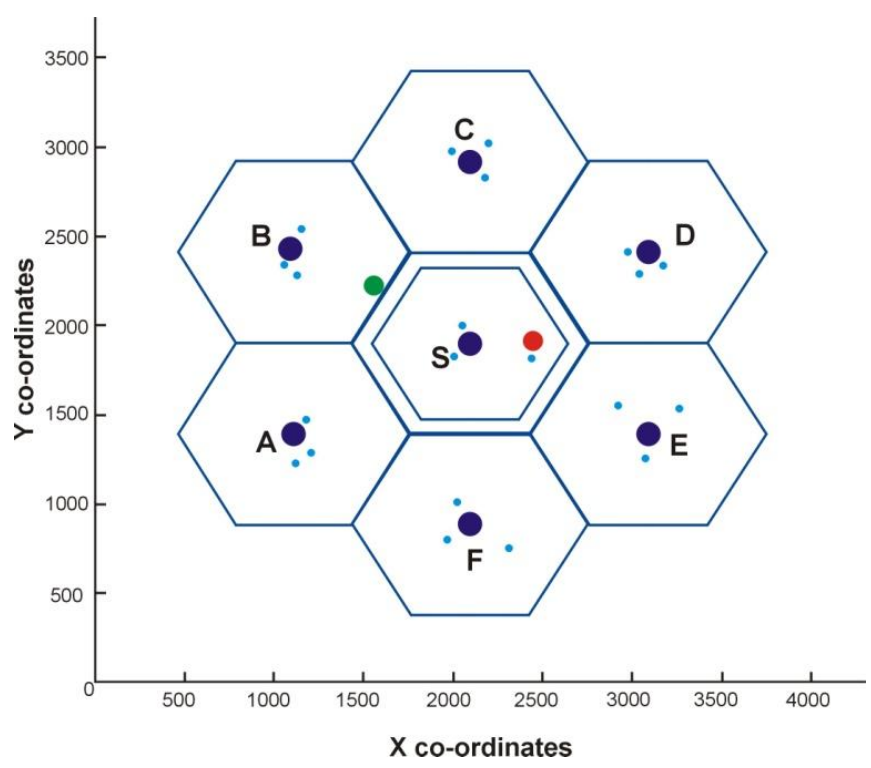

Fig 5: Simulation Scenario using different speed

\subsection{Packet Loss Ratio (PLR)}

Packet Loss affects on the quality of application. It causes by several reasons like errors in wireless network, due to noise, MS receives low RSS from its SBS, congestion in network when the channel becomes overloaded, etc. PLR should be minimum to keep the successful delivery of high QoS. The packet loss value should be preserved at minimum level according to International Telecommunication Union (ITU). Equation 4 shows how to calculate the Packet Loss Ratio[17] during $\mathrm{HO}$ process.

$$
P L R=\frac{\sum_{i} \text { Packets Lost }}{\sum_{i} \text { Packets Sent }} \times 100
$$

where $\mathrm{i}$ is the number of MSs on the service area.

\section{SIMULATION RESULTS and DISCUSSION}

The HO scheme performance evaluation was done using the IEEE802.16e model implemented in MATLAB R2013b Simulator. So, to analyze the latency in HO, throughput and packet loss ratio in WiMAX network, a simulation environment for WiMAX was setup as per the methodology explained before.

\subsection{HO Delay Analysis}

As was mentioned in section 2, the simulation is used to simulate the overall HO time, include the NTAP time and AHOP time. In comparison with conventional Mobile WiMAX scheme where the MS executes the activities of scanning and synchronization with all advertised NBSs which take more times, in proposed system the overall NTAP delay is much reduced because of less scanning activities performed by MS. However, in conventional HO scheme, a large amount of time is wasted for HO preparation especially during the prior AHOP time, namely, ranging, capability negotiation, synchronization and authorization between the MS and selected TBS. So, notice the MS of the final TBS before the MS and the SBS together go for the HO. The SBS exchanges a significant number of MAC messages with the MS during HO preparation time with all PTBSs to ensure that the MS would receive a relevant resources from TBS (its next SBS) after HO. 
The results of simulation show that, the proposed scheme can reduce the NTAP delay by approximately $45 \%$ as shown in Figure 6. Also, as shown in Figure 7, the overall HHO delay in proposed scheme, the reduction by approximately 35\% compared to the conventional Mobile WiMAX scheme. Both NTAP and AHOP delay analysis executed for different speeds MS from $5-120 \mathrm{~km} / \mathrm{hr}$.

\subsection{Throughput Analysis}

Throughput is a measure of how many units of information or number of packets successfully delivered through the network in a given amount of time. The calculation of throughput was done through the $\mathrm{HO}$ process (NTAP+AHOP), where the number of MSs in each cell take into consideration. So, whenever increased the speed of MS, the throughput is increased, because of the MS complete most of the $\mathrm{HO}$ activities in ZE and before enters ZD to avoid throughput degradation or call drop because of the poor of RSS. The average of throughput has taken based on the number of MSs in each cell. The calculation of throughput was done by equation (3).

The graph between throughput Vs speed of MSs is shown in Figure 8. It depicts that as the speed of MSs increases, throughput increases as well. The results of simulation show that, the proposed scheme can increase the throughput by comparison with conventional $\mathrm{HO}$ scheme.

\subsection{Packet Loss Ratio (PLR) Analysis}

Packet Loss Ratio (PLR) indicates the number of packets ratio lost during transmission from source to destination in the $\mathrm{HO}$ activities interval. It is in fact the measure of number of packets lost or undelivered in the network.PLR is calculated from equation (4). The graph is drawn between the PLR and speed of MSs shown in Figure 9. It shows that if there keep on increasing the speed of MSs, then the value of PLR decreases but it finally becomes almost constant after $90 \mathrm{~km} / \mathrm{hr}$. As we mentioned in throughput analysis, whenever increased the speed of MS, the PLR is decreased, because of the MS complete most of the $\mathrm{HO}$ activities in ZE and before enters ZD to avoid excessive packet loss or call drop because of the poor of RSS. The average of PLR has taken based on the number of MSs in each cell. The results of analysis shows that as the speed of MSs increases, PLR decreased as well. The results of simulation show that, the proposed scheme can decrease the PLR by comparison with conventional HO scheme.

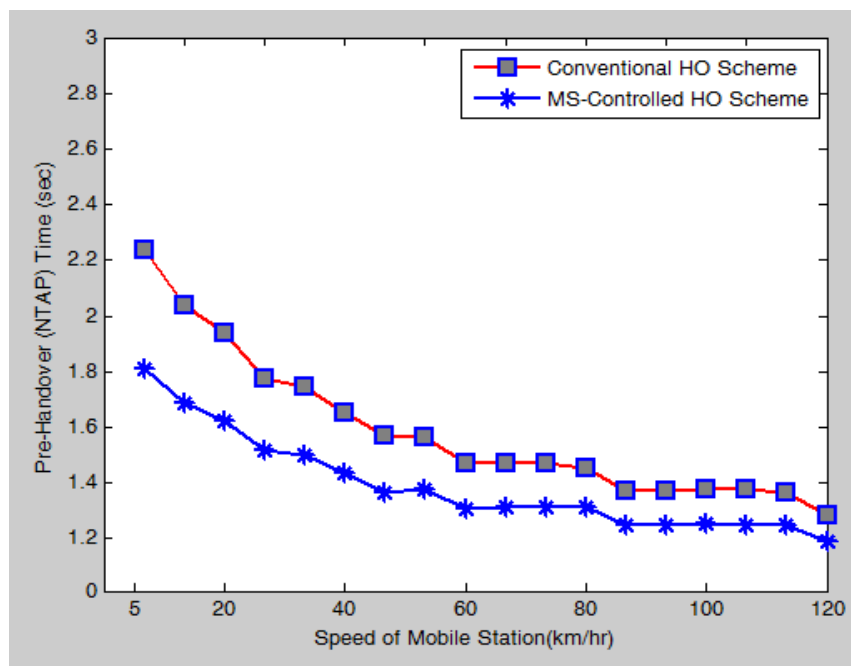

Fig6: Pre-Handover (NTAP) Time versus Speed of MSs

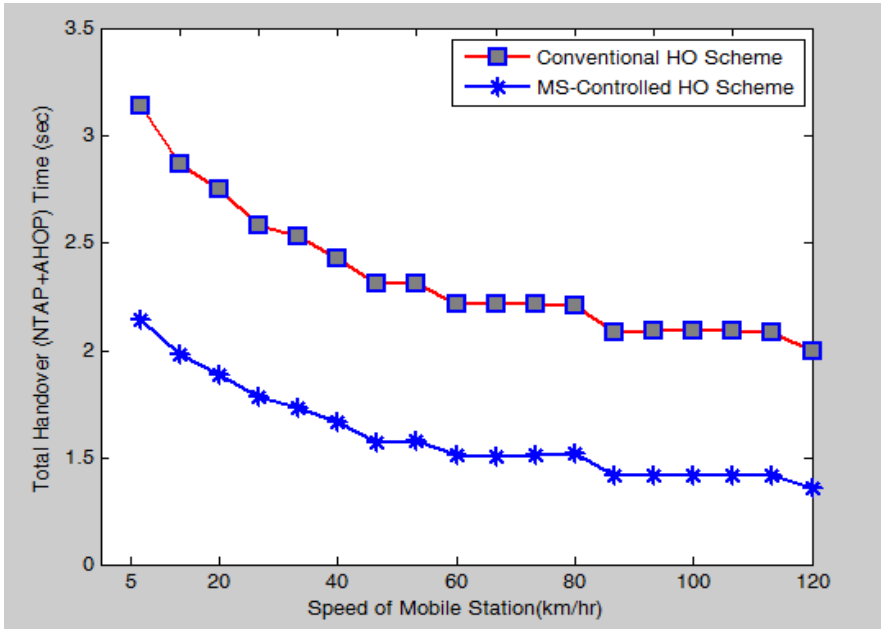

Fig 7: Actual Handover (AHOP) Time versus Speed of MSs

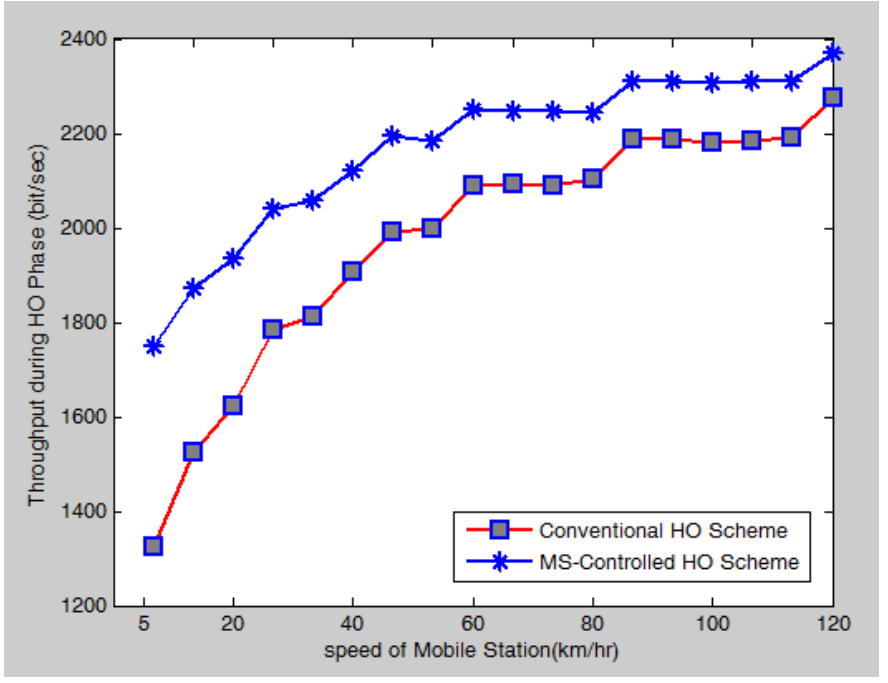

Fig 8: Throughput versus Speed of MSs

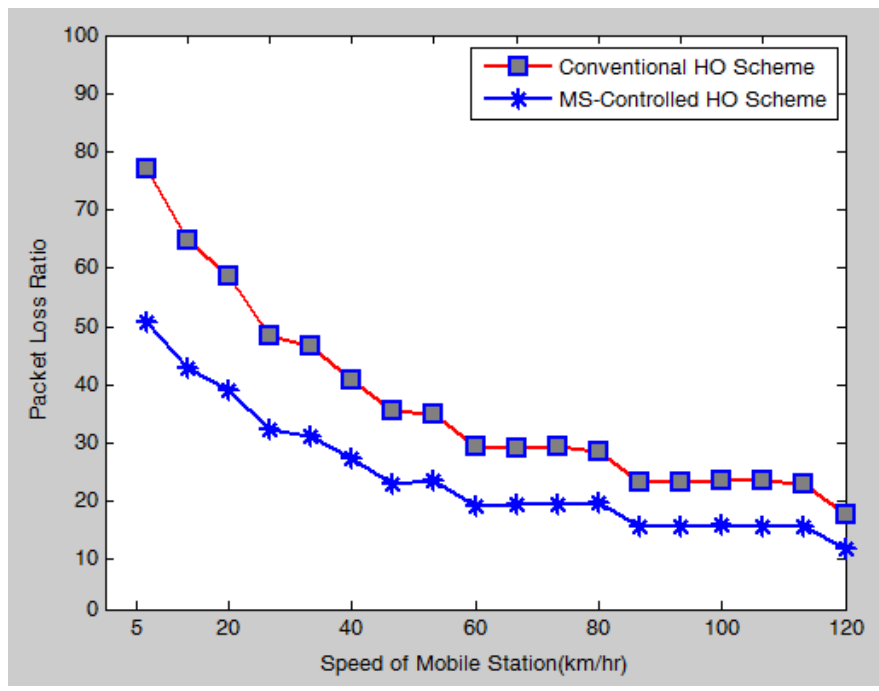

Fig 9: Packet Loss Ratio versus Speed of MSs 


\section{CONCLUSIONS}

The handover process is essential for any WiMAX broadband wireless communication. Processing delays caused by this process is extremely important. Therefore, in this research we have been described the MS-controlled MAC-layer scheme for accomplishing a fast HHO in Mobile WiMAX networks, based on distance estimation by using RSS and relative AOD-based lookahead technique. It is a clear there a reduction in the overall HO delay due to the smart management by the MS in the two main phases in the Mobile WiMAX HHO procedure, namely, NTAP and AHOP. Aided by the concept of zones, where a good part of $\mathrm{HO}$ procedure is completed before the RSS from SBS reaches the level of HO-threshold. In addition, reduced the scanning overhead due to smart short-listing of NBSs as PTBSs. Furthermore, the distances estimation of PTBSs from MS by using RSS and the lookahead technique based on AOD from line of motion of the MS has enabled good selection of the TBS, accordingly allowing each BS to serve a much larger number of MSs.

Also, we have done a measurement a number of QoS parameters, including throughput and packet loss ratio. Our paper helps in analyzing different fundamental WiMAX QoS parameters for determining the performance of a WiMAX network during $\mathrm{HO}$ procedure. A reduction in the PLR is achieved by implemented the proposed scheme by comparison with conventional HO scheme, whereas a very high average value of throughput is obtained.

\section{REFERENCES}

[1] IEEE 802.16e-2005: IEEE Standard for Local and Metropolitan Area Networks-Part 16: Air Interface for Fixed and Mobile Broadband Wireless Access Systems.

[2] WiMAX Forum White Paper: Mobile WiMAX-Part I, A Technical Overview and Performance Evaluation, August 2006.

[3] Ray S, Ray S,Pawlikowski K, McInnes A, Sirisena H. 2010A Fast and Simple Scheme for Mobile StationControlled Handover in Mobile WiMAX. In Proc. of 5th International Conference on Access Networks;. 3244;Budapest, Hungary.

[4] Ray S, Kumar J, Sen S, Nath J. 2007 Modified distance vector routing scheme for a MANET. The National Conference on Communications. 197-202; Kanpur.

[5] Ray S, Pawlikowski K, Sirisena H. 2010Handover in mobile WiMAX networks: The state of art and research issues. Communications Surveys and Tutorials, IEEE. 12(3):376-399.
[6] Rouil R, Golmie N. Adaptive Channel Scanning for IEEE 802.16e. In: IEEE Military Communications Conference. 2006; 1-6; Washington.

[7] Daniel K., Rohde S, Subik S, Wietfeld C."Performance Evaluation for Mobile WiMAX Handover with a Continuous Scanning Algorithm", In: IEEE Mobile WiMAX Symposium, California 2009, pp. 30-35. IEEE Press.

[8] Ray S, Pawlikowski K, Sirisena H. 2009A fast MAClayer handover for an IEEE 802.16 e-based WMAN. In AccessNets.102-117; Berlin Heidelberg.

[9] Zhang Z, Pazzi R, Boukerche A, Landfeldt B. 2010 Reducing handoff latency for WiMAX networks using mobility patterns. In Wireless communications and networking conference (WCNC), IEEE;1-6;Sydney.

[10] Choi S, Hwang G, Kwon T, Lim A, Cho D. H., Fast handover scheme for realtime downlink services in IEEE 802.16e BWA system, In: IEEE Vehicular Technology Conference,Stockholm 2005, pp. 2028-2032. IEEE Press.

[11] Choi Y. H. 2008 Mobility management of IEEE 802.16 e networks. IJCSNS,8(2):89-93.

[12] Ray S, Pawlikowski K, McInnes A, Sirisena H. SelfTracking Mobile Station Controls Its Fast Handover in Mobile WiMAX", In: IEEE Wireless Communications and Networking Conference, Sydney 2010, pp.1-6. IEEE Press.

[13] Andrews J, Ghosh A, Muhamed R2007 "Fundamentals of WiMAX". Prentice-Hall, Englewood Cliffs.

[14] Bshara M, Deblauwe N, Biesen L., Localization in WiMAX networks based on signal strength observations, In: IEEE Global Communications Conference, New Orleans 2008, IEEE Press.

[15] Camp T, Boleng J., Davies V, 2002"A survey of mobility models for Ad-Hoc network research", J. Wireless Communications and Mobile Computing, 2(5), pp. 483-502.

[16] WiMAX Forum Mobile System Profile, Release 1.0 Approved System Specification,WiMAX Forum Working Group.

[17] Mehta V, Gupta N., May 2012 Performance Analysis of QoS Parameters for WiMAX Networks, International Journal of Engineering and Innovative Technology, 1(5), 105-110. 